\title{
Effects of Surface Composition on the Aerosolisation and Dissolution of Inhaled Antibiotic Combination Powders Consisting of Colistin and Rifampicin
}

\author{
Wenbo Wang, ${ }^{1}$ Qi Tony Zhou, ${ }^{2,3}$ Si-Ping Sun, ${ }^{2}$ John A. Denman, ${ }^{4}$ Thomas R. Gengenbach, ${ }^{5}$ Nicolas Barraud, ${ }^{6,7}$ \\ Scott A. Rice, ${ }^{6,8}$ Jian Li, ${ }^{9}$ Mingshi Yang, ${ }^{1,10}$ and Hak-Kim Chan ${ }^{2,10}$
}

Received 7 August 2015; accepted 15 November 2015; published online 24 November 2015

\begin{abstract}
Colistin is often the only effective antibiotic against the respiratory infections caused by multidrug-resistant Gram-negative bacteria. However, colistin-resistant multidrug-resistant isolates have been increasingly reported and combination therapy is preferred to combat resistance. In this study, five combination formulations containing colistin (COL) and rifampicin (RIF) were prepared by spray drying. The lowest minimum inhibitory concentration (MIC) value against Pseudomonas aeruginosa PAO1 was measured for the formulation of $\mathrm{COL} / \mathrm{RIF}=4: 1$ with relatively high emitted doses (over $80 \%$ ) and satisfactory fine particle fractions (over 60\%). Data from X-ray photoelectron spectroscopy (XPS) and nano-time-of-flight secondary ion mass spectrometry (ToF-SIMS) showed the surfaces of particles were mainly covered by rifampicin even for the formulation with a mass ratio of $\mathrm{COL} / \mathrm{RIF}=4: 1$. Because colistin is hygroscopic and rifampicin is hydrophobic, moisture absorption of combination formulations was significantly lower than the pure colistin formulation in the dynamic vapour sorption results. To investigate the dissolution characteristics, four dissolution test methods (diffusion Franz cell, modified Franz cell, flow-through and beaker methods) were employed and compared. The modified Franz cell method was selected to test the dissolution behaviour of aerosolised powder formulations to eliminate the effect of membrane on dissolution. The results showed that surface enrichment of hydrophobic rifampicin neither affected aerosolisation nor retarded dissolution rate of colistin in the combination formulations. For the first time, advanced surface characterisation techniques of XPS and ToF-SIMS have shown their capability to understand the effect of surface composition on the aerosolisation and dissolution of combination powders.
\end{abstract}

KEYWORDS: colistin; combination antibiotics; dry powder inhaler; respiratory infection; rifampicin.

Wenbo Wang and Qi Tony Zhou contributed equally to this work. Electronic supplementary material The online version of this article (doi:10.1208/s12248-015-9848-z) contains supplementary material, which is available to authorized users.

${ }^{1}$ Department of Pharmacy, University of Copenhagen, Universitetsparken 2, 2100, Copenhagen, Denmark.

${ }^{2}$ Advanced Drug Delivery Group, Faculty of Pharmacy, The University of Sydney, Sydney, NSW 2006, Australia.

${ }^{3}$ Department of Industrial and Physical Pharmacy, College of Pharmacy, Purdue University, West Lafayette, Indiana 47907-2091, USA.

${ }^{4}$ Ian Wark Research Institute, University of South Australia, Mawson Lakes, South Australia 5095, Australia.

${ }^{5}$ CSIRO Manufacturing, Bayview Avenue, Clayton, Victoria 3168, Australia.

${ }^{6}$ Centre for Marine Bio-Innovation and School of Biotechnology and Biomolecular Sciences, The University of New South Wales, Sydney, NSW 2052, Australia.

${ }^{7}$ Department of Microbiology, Genetics of Biofilms Unit, Institut Pasteur, Paris, France.

${ }^{8}$ Singapore Centre for Environmental Life Sciences Engineering, and the School of Biological Sciences, Nanyang Technological University, Singapore, Singapore.
${ }^{9}$ Drug Delivery, Disposition and Dynamics, Monash Institute of Pharmaceutical Sciences, Monash University, Parkville, Victoria, Australia.

${ }^{10}$ To whom correspondence should be addressed. (e-mail: my@farma.ku.dk; kim.chan@sydney.edu.au)

Abbreviations AMU, Atomic mass unit; CF, Cystic fibrosis; COL, Colistin; DPIs, Dry powder inhalers; DVS, Dynamic vapour sorption; ED, Emitted dose; FPF, Fine particle fraction; HPLC, High-performance liquid chromatography; $\mathrm{LB}_{10}$, Luria Bertani medium with $10 \mathrm{~g} /$ $\mathrm{L} \mathrm{NaCl}$; LMIG, Liquid metal ${ }^{79+} \mathrm{Au}$ primary ion gun; MDR, Multidrug resistant; MIC, Minimum inhibitory concentration; OD600, Optical density at $600 \mathrm{~nm}$; RH, Relative humidity; RIF, Rifampicin; SEM, Scanning electron microscopy; SLF, Simulated lung fluid; ToF-SIMS, Nano-time-of-flight secondary ion mass spectrometry; XPS, X-ray photoelectron spectroscopy; XRPD, Xray powder diffraction 


\section{INTRODUCTION}

Respiratory infections are one of the major global health problems. Lower respiratory infections are among the top five leading causes of death worldwide over the last decade (1). Due to only limited disposition of most antibiotics at the infection site after systemic administration, lower respiratory infections are generally difficult to treat (2). Consequently, parenteral antibiotic treatments with high doses are necessary to generate and maintain drug concentrations above minimum inhibitory concentrations (MIC) at the infection sites in airways. However, systemic exposure of high-dose antibiotics can result in severe toxicities and adverse effects in some cases. For example, colistin has been used as the last-resort therapy for respiratory infections caused by multidrugresistant (MDR) Gram-negative "superbugs" but can cause severe dosing-limiting nephrotoxicity in up to $60 \%$ of treated patients (3). There is a strong desire to design a highly efficient delivery system for such toxic but life-saving antibiotics like colistin with a purpose to improve therapeutic efficacy and to reduce adverse effects.

Pulmonary drug delivery systems have attracted increasing interests for the treatment of respiratory infections in the past decade (4). As drugs can be delivered directly to the respiratory tracts, local drug concentration in the respiratory tracts can reach well above the MIC with minimal systemic exposure, which has been evidenced by many preclinical and clinical studies $(5,6)$. In a recent clinical pharmacokinetic study, Yapa et al. has shown that compared with the intravenous route, significantly higher colistin concentrations in the sputum $\left(C_{\max }\right.$ 4-16 mg/L) can be achieved for a prolonged period (colistin concentrations $>3 \mathrm{mg} / \mathrm{L}$ for $12 \mathrm{~h}$ ) by nebulised colistin methanesulphonate solution (4 million IU) in cystic fibrosis (CF) patients; importantly, the systemic drug exposure was negligible $\left(C_{\max }<0.5 \mathrm{mg} / \mathrm{L}\right)(7)$. Currently, only a limited number of inhaled antibiotic products have been approved for treatment of respiratory infections in CF patients (8).

Dry powder inhalers (DPIs) are advantageous over the traditional nebulisers as DPIs are generally more portable, more chemically stable and easier to use (9); albeit administration of DPIs may cause more patient error due to misuse of the different inhalers $(10,11)$. Since only fine particles with aerodynamic diameters of $1-5 \mu \mathrm{m}$ can efficiently reach the lungs (12), drug particles are jet-milled in the conventional manufacturing process of inhalable powders. Unfortunately, fine powders generated by jet-milling are cohesive with poor flow and dispersion properties, due to their high surface energy (13). In industrial practice, coarse carriers are blended with cohesive drug particles to manufacture dry powder formulations of low-dose therapeutics, but this carrier system can increase the mass and volume of each dose and make inhalers bulky for high-dose medications (2). Therefore, particle engineering is essential to formulate inhalable highdose antibiotics by generating free-flowing and highly dispersible fine drug powders with sparing amounts of inactive excipients. Spray drying has been extensively employed to produce high-efficiency inhalable drug particles. Spray drying is a viable technique with scale-up capability and has been successful employed to manufacture dry powder inhalers of insulin (Exubera ${ }^{\circledR}$, Pfizer), tobramycin (TOBI ${ }^{\circledR}$ podhaler ${ }^{\circledR}$, Novartis) and mannitol (Aridol ${ }^{\circledR}$, Pharmaxis). Particle engineering techniques or strategies by addition of dispersion enhancer (e.g. leucine) $(14,15)$, development of porous particles (i.e. Pulmosphere ${ }^{\circledR}$ technology) and formation of nanomatrix particles $(16,17)$ can also be combined with spray drying to achieve superior formulation performance.

In our previous studies, an inhalable powder formulation of colistin with high aerosol performance (fine particle fraction (FPF) over the recovered dose $>80 \%$ ) was developed by the spray drying approach without any additives (18). The colistin powder was amorphous and absorbed a significant amount of water when exposed to high relative humidity (RH) measured by dynamic vapour sorption (DVS) over the total measurement period of up to $90 \mathrm{~h}$. Combining amorphous colistin with crystalline rifapentine has shown moisture protection effects because the crystalline rifapentine particles acted as a carrier which prevented the contacts between hygroscopic colistin particles (19). Also the powder formulations produced by co-spray-drying colistin with hydrophobic rifampicin (in a mass ratio of 1:1) exhibited enhanced antibacterial property and prevented humidity-induced aerosolisation deterioration (6). The moisture protection effect was attributed to the surface coating with rifampicin (6). However, the effect of changing the ratio of the two drugs (COL/RIF) on the surface coating and aerosolisation has not been to be investigated. Moreover, dissolution behaviour of the spray-dried formulations also requires investigation. In this study, colistin was co-spray-dried with rifampicin at various mass ratios. Surface coverage of the rifampicin coating was characterized by the state-of-the-art ultrasensitive surface characterisation techniques of X-ray photoelectron spectroscopy (XPS) and time-of-flight secondary ion mass spectrometry (ToF-SIMS). Aerosolisation performance was measured and dissolution behaviour of the selected formulation was investigated.

\section{MATERIALS AND METHODS}

\section{Chemicals}

Colistin sulphate (COL) was supplied by Zhejiang Shenghua Biology Co., Ltd. (Hangzhou, Zhejiang, China) and rifampicin (RIF) from Hangzhou ICH Imp \& Exp Co. Ltd. (Hangzhou, Zhejiang, China). High-performance liquid chromatography (HPLC) grade acetonitrile, ethanol and methanol were purchased from Fisher Scientific (Fair Lawn, NJ, USA) and trifluoroacetic acid from Sigma-Aldrich (Castle Hill, New South Wales, Australia).

\section{Bacterial Strain}

Pseudomonas aeruginosa PAO1 was obtained from the American Type Culture Collection (Manassas, VA, USA) and maintained in tryptone soya broth (TSB; Oxoid Australia, Adelaide, SA, Australia) with $20 \%$ glycerol at $-80^{\circ} \mathrm{C}$.

\section{Production of Powder Formulation by Spray Drying}

A Büchi 290 spray drier (Büchi Labortechnik AG, Falwil, Switzerland) was employed to generate composite particles at the following conditions: inlet temperature $60^{\circ} \mathrm{C}$; outlet temperature $38-42^{\circ} \mathrm{C}$; aspirator $40 \mathrm{~m}^{3} / \mathrm{h}$; atomizer 
setting $742 \mathrm{~L} / \mathrm{h}$ (flow meter $60 \mathrm{~mm}$ ); feed rate $2 \mathrm{~mL} / \mathrm{min}$. Feed solutions $(6 \mathrm{mg} / \mathrm{mL})$ were prepared by dissolving colistin and/ or rifampicin (at the mass ratio of $4: 1,1: 1$ or 1:4 for the combinations) in a co-solvent of water and ethanol $(1: 1, v / v)$. The spray-dried product was stored in a desiccator with silica gel at $20 \pm 3^{\circ} \mathrm{C}$.

\section{Minimal Inhibitory Concentration Against $P$. aeruginosa}

$P$. aeruginosa can cause both acute and chronic respiratory infections (20); hence, it was selected to evaluate the antibacterial activity of the formulations. $P$. aeruginosa cultures were grown overnight in $3 \mathrm{~mL}$ Luria Bertani medium with $10 \mathrm{~g} / \mathrm{L}$ $\mathrm{NaCl}\left(\mathrm{LB}_{10}\right)$ incubated at $37^{\circ} \mathrm{C}$ with shaking. The broth microdilution method was employed to measure the MICs against $P$. aeruginosa PAO1. An aliquot of an overnight culture was diluted 200 times to an optical density measured at a wavelength of $600 \mathrm{~nm}\left(\mathrm{OD}_{600}\right)$ of $0.005 \mathrm{in} \mathrm{M} 9$ minimal medium (containing $48 \mathrm{mM} \mathrm{Na}_{2} \mathrm{HPO}_{4}, 22 \mathrm{mM} \mathrm{KH} \mathrm{PO}_{4}, 9 \mathrm{mM} \mathrm{NaCl}$, $19 \mathrm{mM} \mathrm{NH}_{4} \mathrm{Cl}, 2 \mathrm{mM} \mathrm{MgSO}_{4}, 5 \mathrm{mM}$ glucose, $100 \mu \mathrm{M} \mathrm{CaCl}_{2}$, $\mathrm{pH} 7.0$ ) giving approximately $10^{6} \mathrm{cfu} / \mathrm{mL}$ for the inoculum (21). Drug concentrations of $0.5,1,2,4,8,16,32$ and $64 \mathrm{mg} / \mathrm{L}$ were obtained by diluting the stock drug solution $(5.12 \mathrm{mg} / \mathrm{mL})$ with fresh M9 medium. The MIC of the combinations was determined by simple mixing of aliquots of the single components. Microplates were incubated at $37^{\circ} \mathrm{C}$ in a humidified incubator for approximately $20 \mathrm{~h}$. MICs were determined $(n=4)$ by measuring $\mathrm{OD}_{600}$ of supernatant directly from the wells in 96-well plates using a microplate reader (Wallac Victor ${ }^{2}$, PerkinElmer, Waltham MA, USA). MIC values were determined as the minimum concentration of treatment that inhibited growth by more than $90 \%$ after $24 \mathrm{~h}$ of incubation (21).

\section{Particle Size}

Scanning electron microscopy and image analysis (SEM, TM3030 Tabletop Microscope, HITACHI, USA) (22) was used as a direct particle sizing method (23). The sample was sputter-coated with gold and the images were captured at $15 \mathrm{kV}$. Martin's diameter of 200 randomly selected particles was determined in a fixed direction (up to down) from SEM images and particle size was measured by the software of the SEM instrument. $\mathrm{D}_{10}, \mathrm{D}_{50}$ and $\mathrm{D}_{90}$, which are diameters at 10,50 and $90 \%$ cumulative number, were calculated from the size distribution data. The broadness of size distribution (span) equals $\left(\mathrm{D}_{90}-\mathrm{D}_{10}\right) / \mathrm{D}_{50}$.

\section{Particle Morphology}

SEM (Carl Zeiss SMT AG, Oberkochen, Germany) was applied to examine particle morphology of the powder formulations. Each sample was spread on a carbon sticky tape and mounted on an SEM stub, followed by sputter coating with gold (15 nm thick) using a K550X sputter coater (Quorum Emitech, Kent, UK). The images were captured at $5 \mathrm{kV}$.

\section{Crystallinity}

Powder crystallinity was evaluated by X-ray powder diffraction (XRPD) (Shimadzu XRD-6000, Shimadzu
Corporation, Kyoto, Japan). $\mathrm{Cu}-\mathrm{K} \alpha$ radiation was used at a voltage of $40 \mathrm{kV}$ and a current of $30 \mathrm{~mA}$. Data were collected at a scan speed of $2^{\circ}$ per min in the range of $5-35^{\circ}$.

\section{Dynamic Water Vapour Sorption}

Moisture sorption behaviour was measured by a dynamic vapour sorption system (DVS-1, Surface Measurement Systems Ltd., London, UK). Each sample was analysed in the measurement chamber at the RH ranging from 0 to $90 \%$. The environmental $\mathrm{RH}$ was increased at $10 \% \mathrm{RH}$ increments from 0 to $90 \%$ for the sorption cycle and then decreased from 90 to $0 \%$ for the desorption cycle. Equilibrium moisture content at each testing $\mathrm{RH}$ was determined by a $\mathrm{dm} / \mathrm{dt}$ of $0.002 \%$ per minute (18).

\section{X-Ray Photoelectron Spectroscopy}

X-ray photoelectron spectroscopy (XPS) analysis was performed using an AXIS Ultra DLD Spectrometer (Kratos Analytical Ltd., Manchester, UK), equipped with a monochromated Al $\mathrm{K} \alpha$ source at a power of $140 \mathrm{~W}$ $(10 \mathrm{~mA}, 14 \mathrm{kV})$. A small quantity of each sample was filled into shallow wells of custom-made powder sample holders (24). Charging of the samples during irradiation was compensated for by the inbuilt charge neutraliser. A reference binding energy of $285.0 \mathrm{eV}$ for the aliphatic hydrocarbon $\mathrm{C} 1 \mathrm{~s}$ component was used to correct for any remaining offsets due to charge neutralisation of specimens under irradiation (25). The pressure in the main vacuum chamber during analysis was typically $10^{-6} \mathrm{~Pa}$. Spectra were recorded with the nominal photoelectron detection normal to the sample surface. Note, however, that in the case of powders of random orientations, the microscopic emission angle is ill-defined. As a consequence, the sampling depth might vary between 0 and 5-10 $\mathrm{nm}$ depending on the kinetic energy of the measured photoelectrons. The area analysed on each sample had approximate dimensions $0.3 \mathrm{~mm} \times 0.7 \mathrm{~mm}$. The elemental composition of the samples was obtained from survey spectra (160 eV pass energy) using sensitivity factors supplied by the manufacturer. High-resolution spectra of individual peaks were recorded at $20 \mathrm{eV}$ pass energy which results in a peak width (full width at half maximum) of typically $0.9-1.1 \mathrm{eV}$ for organic polymeric materials.

In order to determine relative fractions of colistin and rifampicin on the surface of the combination particles, reference data were acquired from the two pure compounds. The data obtained from the combination particles were then compared to the reference data and fractions of the pure compounds estimated as follows. The atomic concentration of each of the elements present $(\mathrm{C}, \mathrm{O}, \mathrm{N}$ and $\mathrm{S})$ in the combination particles was assumed to be a linear combination of the corresponding concentrations in the pure compounds, appropriately scaled and normalised using the number of respective atoms in one molecule of colistin or rifampicin. This method is particularly reliable in cases where the concentrations in the pure compounds are very different $(\mathrm{N}$ and $\mathrm{S}$ ) but rather unreliable in cases where concentrations are similar ( $\mathrm{C}$ and $\mathrm{O}$ ). However, in the case of $\mathrm{C}$ and $\mathrm{O}$, the highresolution 1s spectra for the two compounds are very different (as are the $\mathrm{N}$ 1s spectra) because of the different 
chemical structures. This allows the surface fractions of colistin and rifampicin in the combination particles to be estimated using curve fitting: the reference spectra of colistin and rifampicin can be used as two model-fit components to calculate optimised curve fits of the spectra of the combination particles. This yields the relative number of either $\mathrm{C}$, or $\mathrm{O}$ or $\mathrm{N}$ atoms which are present as part of either colistin or rifampicin (24). Scaled using the respective number of $\mathrm{C}$ or $\mathrm{O}$ atoms in one molecule of the pure compound, these fractions can be converted to relative molecular fractions of colistin or rifampicin.

\section{Time-of-Flight Secondary Ion Mass Spectrometry}

ToF-SIMS experiments were performed using a PHI TRIFT V nanoTOF instrument (Physical Electronics Inc., Chanhassen, MN, USA) equipped with a pulsed liquid metal ${ }^{79+} \mathrm{Au}$ primary ion gun (LMIG), operating at $30 \mathrm{keV}$ energy. Dual charge neutralisation was provided by an electron flood gun and $10 \mathrm{eV} \mathrm{Ar}^{+}$ions. Surface analyses were performed using "unbunched" $\mathrm{Au}_{1}$ instrument settings to optimise spatial resolution. Raw data were collected in the positive SIMS mode at a number of locations typically using a $50 \times$ $50 \mu \mathrm{m}$ raster area, with 4 min acquisitions.

Analyses of colistin and rifampicin standards identified characteristic peak fragments for use in mapping the components in the combination powders. Peaks corresponding to the protonated molecular ion signal for each antibiotic were of low intensity; hence, higher intensity characteristic fragments were used instead. For colistin, the sum of peaks at $\mathrm{m} / \mathrm{z}$ $\sim 30$ atomic mass unit (amu) and $\sim 86 \mathrm{amu}$ was selected, corresponding to $\mathrm{CH}_{4} \mathrm{~N}^{+}$and $\mathrm{C}_{5} \mathrm{H}_{12} \mathrm{~N}^{+}$fragments, respectively. For rifampicin, $m / z \sim 99$ amu was selected, corresponding to the methylated piperazine fragment $\left(\mathrm{C}_{5} \mathrm{H}_{11} \mathrm{~N}_{2}{ }^{+}\right)$. Sample spectra and images were processed using WincadenceN software (Physical Electronics Inc., Chanhassen, MN, USA).

\section{In Vitro Aerosol Performance}

In vitro aerosolisation performance was evaluated by a next-generation impactor (Apparatus X, USP X, Copley, Nottingham, UK) with a USP induction port (USP throat). Seven impactor stages and the filter were coated by MOLYKOTEÒ 316 silicone grease release spray (Dow Corning Corp., Midland, MI) before each testing to minimise particle bounce. Each capsule (size 3 hydroxypropyl methylcellulose capsules, Capsugel, West Ryde, Australia) was loaded with $10 \pm 1 \mathrm{mg}$ of powder and dispersed through an Osmohaler ${ }^{\circledR}$ in a controlled environment cabinet: temperature, $20 \pm 3{ }^{\circ} \mathrm{C}$ and relative humidity, $60 \pm 3 \%$. A standard pharmacopeial dispersion procedure was used (USP 38), whereby $4 \mathrm{~L}$ of air was passed through the inhaler at an airflow of $100 \mathrm{~L} / \mathrm{min}$ for $2.4 \mathrm{~s}$, with a pressure drop of $<4 \mathrm{kPa}$ (approximately $3.2 \mathrm{kPa}$ at $100 \mathrm{~L} / \mathrm{min}$ ) across the device $(26,27)$. The Osmohaler® has a similar design and resistance to the Aerolizer® (see Supplement 1). It was used here as a model device because it has a low resistance and patients can comfortably generate sufficient flow rates to disperse powder formulations. Three replicates were carried out for each formulation. Drug particles deposited on capsule, inhaler,
USP throat and each stage of the impactor were collected using a co-solvent (acetonitrile/water, 3:7). Emitted dose (ED) was calculated as a percentage of the drug released from the capsules and inhaler device. FPF was calculated as the percentage mass of drug particles with an aerodynamic diameter smaller than $5 \mu \mathrm{m}$ in the emitted dose relative to the total recovered drug.

\section{Dissolution}

Four dissolution methods (diffusion Franz cell, modified Franz cell, beaker method and flow-through cell) were applied in this study for the investigation of a suitable dissolution method for inhalable antibiotics. The medium in all dissolution methods was a simulated lung fluid (SLF) at pH 7.4 (28) with ascorbic acid $(2 \%, w / v)$ in order to prevent oxidation of the drug. Twenty-three milliliters of SLF was used for all the dissolution test methods, which resulted in over 10 times volume of saturation of the medium for all samples to ensure sink conditions. The total volume of epithelial lining fluid is estimated to be $20-40 \mathrm{~mL}$ (29); therefore, $23 \mathrm{~mL}$ of SLF was used. It is noted that the sink conditions may not be realistic to all drugs in vivo but were used in this study to reflect the total volume of epithelial lining fluid. Aerosol particles $(2.5-3 \mathrm{mg}$ ) were aerosolised as described in the "Aerosol Performance" section and collected on a paper filter (Whatman ${ }^{\circledR}$ Grade 2 , pore size $5 \mu \mathrm{m}$, GE Healthcare, Parramatta, Australia) under the dispersion jets in the stage 4 of a NGI. Stirrer speed was set to $500 \mathrm{rpm}$ for the two Franz cell methods and the beaker method. Medium temperature was kept at $37^{\circ} \mathrm{C}$ for all the systems. An aliquot of $0.5 \mathrm{~mL}$ sample was taken at selected time intervals $(5,10$, $15,20,25,30,45,60,90,120$ and $180 \mathrm{~min})$ and $0.5 \mathrm{~mL}$ of fresh medium was added immediately after sample collection. Details of each method are described below.

\section{Diffusion Franz Cell and Modified Franz Cell Methods}

These two methods were determined using Franz dissolution cells in a heated station stirrer (V6B, PermeGear Inc., Bethlehem, USA). The cell reservoirs were filled with $23 \mathrm{~mL}$ of SLF, which resulted in a positive meniscus of the surface. For the diffusion Franz cell method, details were described previously (5). The aerosol powder collected on the paper filter from NGI was placed on the top of the Franz cell with the filter paper between the powder and medium. For the modified Franz cell method, the powder was in contact with the medium directly.

\section{Beaker Method}

The beaker method was adopted from the literature (30). After powder collection, the paper filter was placed in the container (44 mm wide $\times 57 \mathrm{~mm}$ high, Livingstone, Australia) with $23 \mathrm{~mL}$ SLF. The contents were then stirred by a magnet at $500 \mathrm{rpm}$.

\section{Flow-Through Cell}

A flow-through cell system was adopted from the previous studies (31). SLF was passed through a $25-\mathrm{mm}$ flow-through cell 
(Millipore Swinnex filter holders, USA) and re-circulated in a closed-loop system by a peristaltic pump at $1 \mathrm{~mL} / \mathrm{min}$.

\section{Drug Quantification}

Concentrations of colistin and rifampicin were determined by an established HPLC method (6). Briefly, the HPLC system consisted of a Shimadzu CBM-20A controller, LC-20AT pump, SIL-20A HT autosampler, SPD-20A UV/ VIS detector (Shimadzu, Kyoto, Japan), and a PhenoSphereNext $5 \mu \mathrm{m}$ C18 $150 \times 4.60 \mathrm{~mm}$ column (Phenomenex, Torrance, CA). The mobile phase consisted of $0.05 \%$ trifluoroacetic acid in Milli-Q water (A) and methanol (B). The gradient program was set as: $30 \% \mathrm{~B}$ to $80 \% \mathrm{~B}$ in $15 \mathrm{~min}$, then $80 \% \mathrm{~B}$ to $30 \% \mathrm{~B}$ in $5 \mathrm{~min}$ with a flow rate of $1 \mathrm{~mL} / \mathrm{min}$. A calibration curve $(0.01-1 \mathrm{mg} / \mathrm{mL})$ was prepared for colistin and rifampicin in a co-solvent (acetonitrile/water, volume ratio $1: 1)$ and was linear in the required concentration range $\left(r^{2}>0.999\right)$.

\section{Statistical Analysis}

One-way analysis of variance (ANOVA) with a Tukey post hoc analysis was employed for statistical analysis with probability values of less than $0.05(p<0.05)$ considered as a statistical significant difference.

Difference factor $\left(f_{1}\right)$ and similarity factor $\left(f_{2}\right)$ were used to evaluate and compare the dissolution profiles (32).

$$
\begin{aligned}
& f_{1}=\frac{\sum_{j=1}^{n}\left|R_{j}-T_{j}\right|}{\sum_{j=1}^{n} R_{j}} \times 100 \% \\
& f_{2}=50 \times \log \left\{\left[1+(1 / n) \sum_{j=1}^{n}\left|R_{j}-T_{j}\right|^{2}\right]^{-0.5} \times 100\right\}
\end{aligned}
$$

A value lower than 15 of $f_{1}$ indicates no evidence of difference and a value higher than 50 of $f_{2}$ indicates similarity and no important difference between dissolution profiles.

\section{RESULTS}

\section{Physico-Chemical Properties}

The content uniformity results (see Supplement 2) showed that the ratios of colistin and rifampicin in the combination formulations were close (within 1\%) to the theoretical values. Table I showed that all spray-dried powder formulations had fine particle sizes with $\mathrm{D}_{50}$ between 1.15 and $1.29 \mu \mathrm{m}$ and $\mathrm{D}_{90}<2.5 \mu \mathrm{m}$.

SEM micrographs (Fig. 1a) showed the spray-dried pure colistin particles exhibited corrugated surfaces. The spraydried pure rifampicin had a flake shape (Fig. 1e). The shape of the combination particles was between wrinkled and flakeshaped (Fig. 1b-d).

XRD data showed that all of the spray-dried powder formulations were amorphous (Supplement 3). Data from previous studies demonstrated that amorphous, pure colistin powder produced by spray drying absorbed significant amount of water (up to $30 \%, w / w$ ) at the elevated humidity (18). In contrast, the water absorption of the pure rifampicin formulation was substantially lower (Fig. 2). There was no significant difference $(p>0.05)$ between the spray-dried COL alone and COL/RIF 4:1, spray-dried RIF alone and COL/RIF 1:4, but there were differences between COL/RIF 1:1, COL/ RIF 4:1 and COL/RIF 1:4. There is a clear trend that increasing the proportion of colistin resulted higher water absorption. For the formulation of COL/RIF 1:4, the absorbed water was less than $10 \%$, even at a high $\mathrm{RH}$ of $80 \%$. Mass change of all formulations was zero at the end of desorption indicating no moisture-induced recrystallisation occurred. A sorption hysteresis was observed for each sample which could be attributable to the slower escape during desorption process of those water molecules trapped in the invaginations of particles (33).

Table II presented the elemental compositions of two pure compounds and three combination formulations measured by XPS. C, N, O and S were the only elements detected by XPS as expected (XPS cannot detect $\mathrm{H}$ ). The measured surface composition of colistin was consistent with its theoretical value. The corresponding compositional values for rifampicin indicated the presence of minor adventitious hydrocarbon contamination (excess of carbon), which is very common in XPS surface analysis. A thin layer of carbonaceous material is usually found on the surface of most airexposed samples. Even small exposures to atmosphere can produce these films. Adventitious carbon is generally comprised of a variety of (relatively short chain, perhaps polymeric) hydrocarbons species with small amounts of both singly and doubly bound oxygen functionality (34). The surface composition of three different composite formulations varied continuously between those of the two constituents, with the relative concentrations of oxygen, nitrogen and sulphur decreasing with an increase in concentration of rifampicin. Based on the measured compositions of colistin and rifampicin (Table II, first and last columns, respectively), we can use the elemental compositions of the composite

\begin{tabular}{|c|c|c|c|c|c|}
\hline & Pure COL & $\begin{array}{l}\mathrm{COL} / \mathrm{RIF} \\
4: 1\end{array}$ & $\begin{array}{l}\mathrm{COL} / \mathrm{RIF} \\
1: 1\end{array}$ & $\begin{array}{l}\mathrm{COL} / \mathrm{RIF} \\
1: 4\end{array}$ & Pure RIF \\
\hline $\mathrm{D}_{10}(\mu \mathrm{m})$ & 0.70 & 0.75 & 0.80 & 0.72 & 0.84 \\
\hline $\mathrm{D}_{50}(\mu \mathrm{m})$ & 1.19 & 1.15 & 1.20 & 1.21 & 1.29 \\
\hline $\mathrm{D}_{90}(\mu \mathrm{m})$ & 1.91 & 1.73 & 2.01 & 2.18 & 2.07 \\
\hline Span & 1.02 & 0.85 & 1.68 & 1.20 & 0.95 \\
\hline $\operatorname{MICs}(\mu \mathrm{g} / \mathrm{mL})$ & 4 & 2 & 4 & 8 & 16 \\
\hline
\end{tabular}

Table I. Particle Size and MICs Against P. aeruginosa PAO1 of the Spray-Dried Antibiotic Formulations 


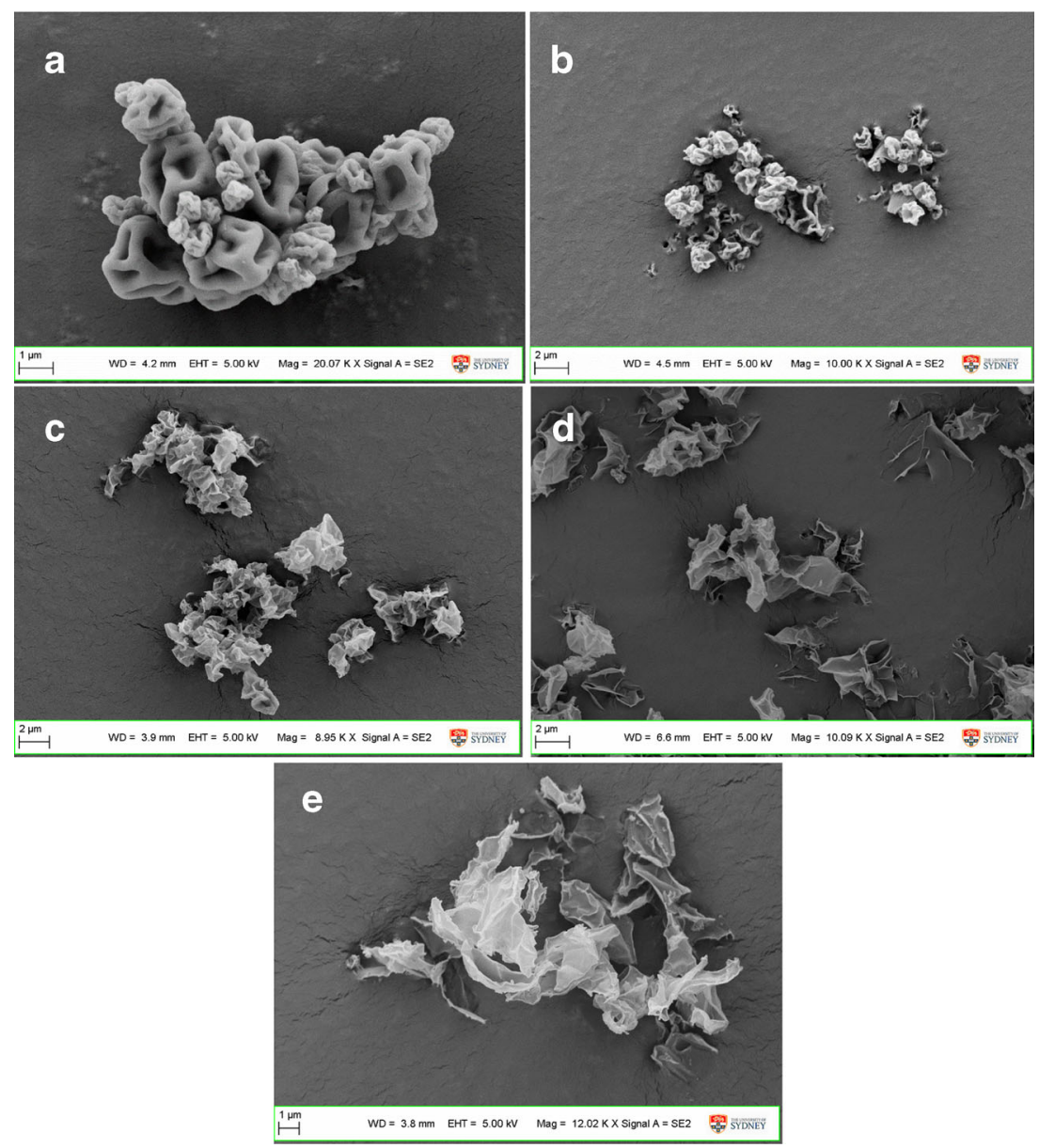

Fig. 1. SEM images of the spray dried formulations: a spray-dried pure colistin; $\mathbf{b}$ co-spray dried COL/RIF = 4:1; $\mathbf{c}$ co-spray dried COL/RIF = 1:1; $\mathbf{d}$ co-spray dried COL/RIF = 1:4; $\mathbf{e}$ spray-dried pure rifampicin

formulations to estimate relative molar fractions of colistin and rifampicin (see "Materials and Methods"). A high proportion of rifampicin on the particle surface (in excess of $90 \%$ ) was achieved by the composite formulation with a mass ratio of $1: 1$, indicating the particle surfaces were dominated by the rifampicin.
These estimated surface fractions were confirmed by employing the second method described in the "Materials and Methods" section, using curve-fitting of highresolution spectra ( $\mathrm{C} 1 \mathrm{~s}, \mathrm{O} 1 \mathrm{~s}$ and $\mathrm{N}$ 1s) using model spectra of colistin and rifampicin as fit components, followed by appropriate scaling according to the number

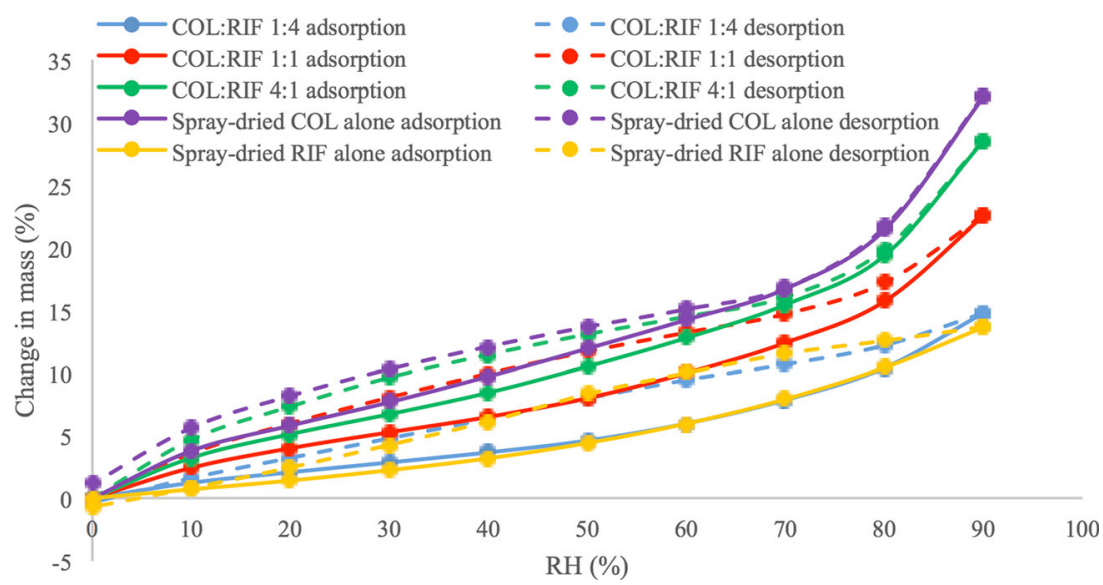

Fig. 2. Dynamic water sorption and desorption behaviour of spray-dried antibiotic formulations 
Table II. Elemental Composition of Colistin (COL), Rifampicin (RIF) and the Composite Powders, as Measured by XPS. Concentrations Are Expressed as Atomic Ratios X/C (Atomic Concentration of Element X Relative to That of C). Mean Values \pm SD $(n=3)$. Also Shown Are Estimates of Molar Fractions of COL and RIF in Composite Formulations, Based on Elemental Compositions (Last Two Rows)

\begin{tabular}{llllll}
\hline & & COL/RIF & COL/RIF & COL/RIF \\
& Pure COL & $4: 1$ & $1: 1$ & $1: 4$ & Pure RIF \\
\hline N/C & $0.277 \pm 0.002$ & $0.137 \pm 0.001$ & $0.107 \pm 0.002$ & $0.097 \pm 0.002$ & $0.087 \pm 0.001$ \\
O/C & $0.349 \pm 0.003$ & $0.286 \pm 0.007$ & $0.266 \pm 0.003$ & $0.264 \pm 0.002$ & $0.269 \pm 0.009$ \\
S/C & $0.040 \pm 0.000$ & $0.011 \pm 0.000$ & $0.005 \pm 0.000$ & $0.002 \pm 0.000$ & - \\
COL & $100.0 \%$ & $13.0 \%$ & $5.0 \%$ & $2.0 \%$ & $0.0 \%$ \\
RIF & $0.0 \%$ & $87.0 \%$ & $95.0 \%$ & $98.0 \%$ & $100.0 \%$ \\
\hline
\end{tabular}

of $\mathrm{C}, \mathrm{O}$ or $\mathrm{N}$ atoms in each molecule. Supplement 4 shows a $\mathrm{C}$ 1s spectrum curve-fitted with model spectra as an example. Table III lists the resulting estimates of the molar fractions of colistin and rifampicin. Variation in estimated fractions between different methods used was observed; this variation mainly reflects the uncertainty associated with curve-fitting. Despite the variation, there is general agreement between the different methods. The curve fit-based values clearly confirm the conclusion that the surface of the COL/RIF composite particles was dominated by rifampicin, even when rifampicin represents only $20 \%$ of the bulk mass.

Figure 3 shows the mapping of colistin (blue) and rifampicin (red) signals in three combination formulations in an area of $50 \times 50 \mu \mathrm{m}$ measured by ToF-SIMS. The distribution of colistin in the pure colistin formulation was shown in Supplement 5. Attributable to the surface sensitivity of ToF-SIMS, the intensity and distribution of the species relates to their abundance within the top 1$2 \mathrm{~nm}$ of the surface. Hence, the data indicated the surface of particles was dominated by rifampicin for the formulations with colistin/rifampicin ratios of $1: 1$ and 1:4. Even with a very low concentration $(\mathrm{COL} / \mathrm{RIF}=4: 1)$ of rifampicin in the formulation, rifampicin signal was abundant, while there was rare signal of colistin in the formulation with a high concentration $(\mathrm{COL} / \mathrm{RIF}=1: 4)$ of rifampicin. However, the coverage of rifampicin on the particle surface may not be complete as there was still small quantity of colistin signal observed in the formulation with high concentration of rifampicin $(\mathrm{COL} / \mathrm{RIF}=1: 4)$. This result was also in good agreement with the XPS data, which confirmed the presence of approximately $2 \%$ of colistin on the particle surfaces in the formulation with approximately $98 \%$ of rifampicin.

\section{Antimicrobial Activities}

The MICs against $P$. aeruginosa of the formulations are presented in Table I. The combination formulation of COL/ $\mathrm{RIF}=4: 1$ had the lower MIC $(2 \mu \mathrm{g} / \mathrm{mL})$ compared to pure colistin and pure rifampicin, thus showing an enhanced antimicrobial activity.

\section{Aerosol Performance}

The aerosol performance of different formulations is shown in Table IV. All the spray-dried formulations achieved relatively high ED over $75 \%$ and high FPF over $64 \%$ for both colistin and rifampicin. There was no significant difference in ED of colistin between the spray-dried colistin alone and the combination formulations of COL/RIF 4:1 and 1:1 $(p>0.05)$. The combination formulation of COL/RIF 1:4 had the highest $\mathrm{ED}$ of colistin among all the formulations $(p<0.05)$. There was no difference in FPF of colistin for all formulations $(p>0.05)$.

Similar trend in ED and FPF was shown for rifampicin. There was no significant difference in ED of rifampicin between the spray-dried rifampicin alone and COL/RIF $1: 4(p>0.05)$ and between the two combination formulations of COL/RIF $1: 1$ and 4:1 $(p>0.05)$. Two formulations with higher rifampicin concentrations, spraydried rifampicin and COL/RIF 1:4 had a significantly higher ED of rifampicin than COL/RIF 1:1 and COL/RIF $4: 1 \quad(p<0.05)$. The combination formulation of $\mathrm{COL} / \mathrm{RIF}$ 1:4 had the highest FPF of rifampicin. The FPF of combination formulation of 1:1 was significantly higher than the spray-dried rifampicin alone and COL/RIF $4: 1$ $(p<0.05)$.

Table III. Estimates of Molar Fractions of Colistin (COL) and Rifampicin (RIF) in Composite Formulations, Based on Curve-Fitting High Resolution Spectra with Model Spectra. See Text for Details

\begin{tabular}{|c|c|c|c|c|c|c|}
\hline & & Pure COL & $\begin{array}{l}\text { COL:RIF } \\
4: 1\end{array}$ & $\begin{array}{l}\text { COL:RIF } \\
1: 1\end{array}$ & $\begin{array}{l}\text { COL:RIF } \\
1: 4\end{array}$ & Pure RIF \\
\hline \multirow[t]{2}{*}{$\mathrm{C} 1 \mathrm{~s}$} & $\mathrm{COL}$ & $100.0 \%$ & $16.8 \%$ & $4.2 \%$ & $0.9 \%$ & $0.0 \%$ \\
\hline & RIF & $0.0 \%$ & $83.2 \%$ & $95.8 \%$ & $99.1 \%$ & $100.0 \%$ \\
\hline \multirow[t]{2}{*}{$\mathrm{O} 1 \mathrm{~s}$} & $\mathrm{COL}$ & $100.0 \%$ & $17.5 \%$ & $8.5 \%$ & $3.6 \%$ & $0.0 \%$ \\
\hline & RIF & $0.0 \%$ & $82.5 \%$ & $91.5 \%$ & $96.4 \%$ & $100.0 \%$ \\
\hline \multirow[t]{2}{*}{$\mathrm{N} 1 \mathrm{~s}$} & $\mathrm{COL}$ & $100.0 \%$ & $11.3 \%$ & $3.2 \%$ & $2.1 \%$ & $0.0 \%$ \\
\hline & RIF & $0.0 \%$ & $88.7 \%$ & $96.8 \%$ & $97.9 \%$ & $100.0 \%$ \\
\hline
\end{tabular}



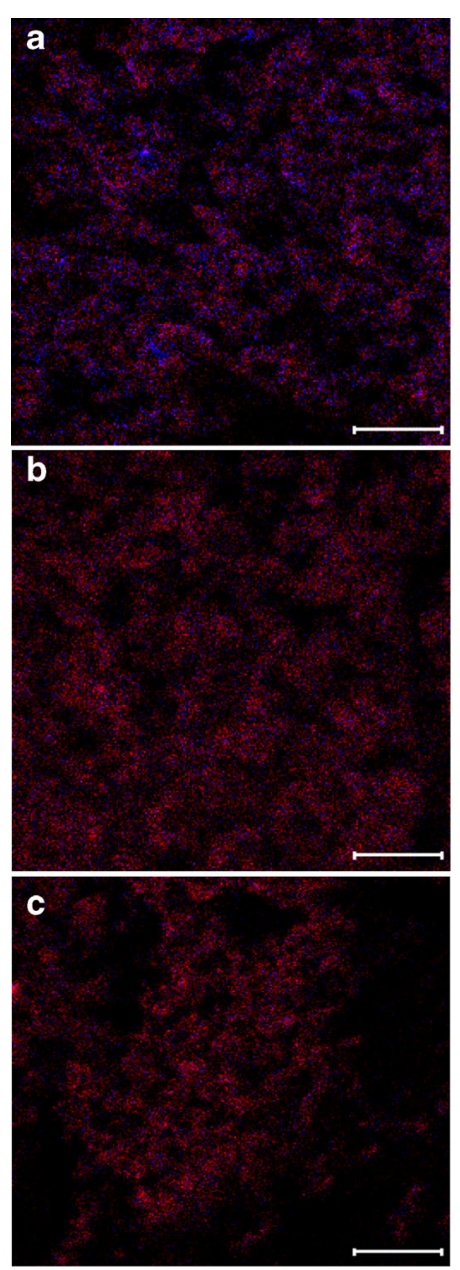

Fig. 3. Distribution of colistin (blue) and rifampicin (red) on the outermost surface of composite particles measured by ToF-SIMS: a COL/RIF 4:1; b COL/RIF 1:1; c COL/RIF 1:4 (scale bar represents $10 \mu \mathrm{m})$

The ED and FPF values of COL/RIF 1:1 formulation via the Osmohaler measured here by an NGI is lower than those previously reported via the Aerolizer measured by a multistage liquid impinger (6). Previous studies showed smaller capsule holes enhanced the de-agglomeration in the modified Aerolizers (35). Here, the Osmohaler created a larger hole in each end of capsule compared to four smaller holes created by the Aerolizer, which may led to lower FPF. Moreover, significantly more drugs deposited on the capsule and
Osmohaler during aerosolisation tests of the present study than those on the Aerolizer in the previous study, which could be attributed to the different inhaler configurations and drugadhesion properties of the inhaler materials (9) (Fig. 4).

\section{Dissolution Profile}

Comparison of Dissolution Methods for Inhalable Antibiotics

The combination formulation of 1:1 was selected to investigate the dissolution of inhalable antibiotics. Dissolution results are shown in Fig. 5 (a, b). To provide detailed information of dissolution data, results of colistin measured by different methods in the first $50 \mathrm{~min}$ is shown in Supplement 6 . The values of $f_{1}$ (difference factor) and $f_{2}$ (similarity factor) were calculated based on the dissolution profiles. For colistin (Fig 5a), there was no difference between the data measured by diffusion Franz cell and modified Franz cell $\left(f_{1}<15\right.$ and $\left.f_{2}>50\right)$. There was also no difference in the results between the flow-through cell and beaker methods. However, dissolution profiles from the beaker method and flow through cell method were statistically faster than those measured by the modified Franz cell and diffusion Franz cell methods in the first $50 \mathrm{~min}\left(f_{1}>15\right.$, Supplement 6). Colistin dissolved very fast in the beaker and flow-through cell measurements with more than $60 \%$ of total drug being dissolved in 5 min compared to around $20-30 \%$ drug release using the modified and diffusion Franz cell. The maximum drug dissolved for beaker method was around 95\% and flowthrough cell method was $89 \%$ within 20 min, while more than 90\% total drug dissolved using modified and diffusion Franz cell within $30 \mathrm{~min}$.

In contrast to colistin, the dissolution rate for rifampicin was considerably lower when quantified using the diffusion Franz cell method (only less than $70 \%$ total drug released in $3 \mathrm{~h})$ or by the modified Franz cell $(80 \%$ total drug released in $45 \mathrm{~min}$ ). There was no statistical difference in the dissolution rate between the flow-through cell and beaker methods $(p>0.05)$. Both methods resulted in a fast drug release (around $60 \%$ of drug was released in $5 \mathrm{~min}$ ). The flowthrough cell method led to a lower total drug release $(80 \%)$ compared to the modified Franz cell and beaker methods.

\section{Effect of Drug Powder Mass on Dissolution}

Two dose ranges of co-spray dried combination powder with a mass ratio of 1:1 were used to investigate the effect of

Table IV. Aerosol Performance of Colistin and Rifampicin: Emitted Dose (ED) and Fine Particle Fraction (FPF) of the Spray-Dried Antibiotic Powder Formulations from the Osmohaler Device (Error Bars Represent Standard Deviation, $n=3$ )

\begin{tabular}{|c|c|c|c|c|}
\hline \multirow[b]{2}{*}{ Formulation } & \multicolumn{2}{|c|}{ ED (Average \% mass deposited) } & \multicolumn{2}{|c|}{ FPF (Average \% mass deposited) } \\
\hline & $\mathrm{COL}$ & RIF & $\mathrm{COL}$ & RIF \\
\hline Spray-dried COL alone & $77.6 \pm 2.1$ & - & $70.4 \pm 2.9$ & - \\
\hline Spray-dried RIF alone & - & $86.6 \pm 0.3$ & - & $68.7 \pm 1.6$ \\
\hline COL:RIF $4: 1$ & $77.7 \pm 1.8$ & $77.1 \pm 1.8^{*}$ & $68.8 \pm 1.3$ & $64.5 \pm 2.5$ \\
\hline COL:RIF 1:1 & $80.7 \pm 0.8$ & $79.4 \pm 1.1^{*}$ & $73.5 \pm 1.0$ & $72.9 \pm 1.2^{*}$ \\
\hline COL RIF 1:4 & $86.7 \pm 1.3^{*}$ & $89.8 \pm 2.1$ & $68.1 \pm 2.3$ & $80.4 \pm 1.6^{*}$ \\
\hline
\end{tabular}

$* p<0.05$ (significant difference compared with the spray-dried pure drug (colistin or rifampicin)) 

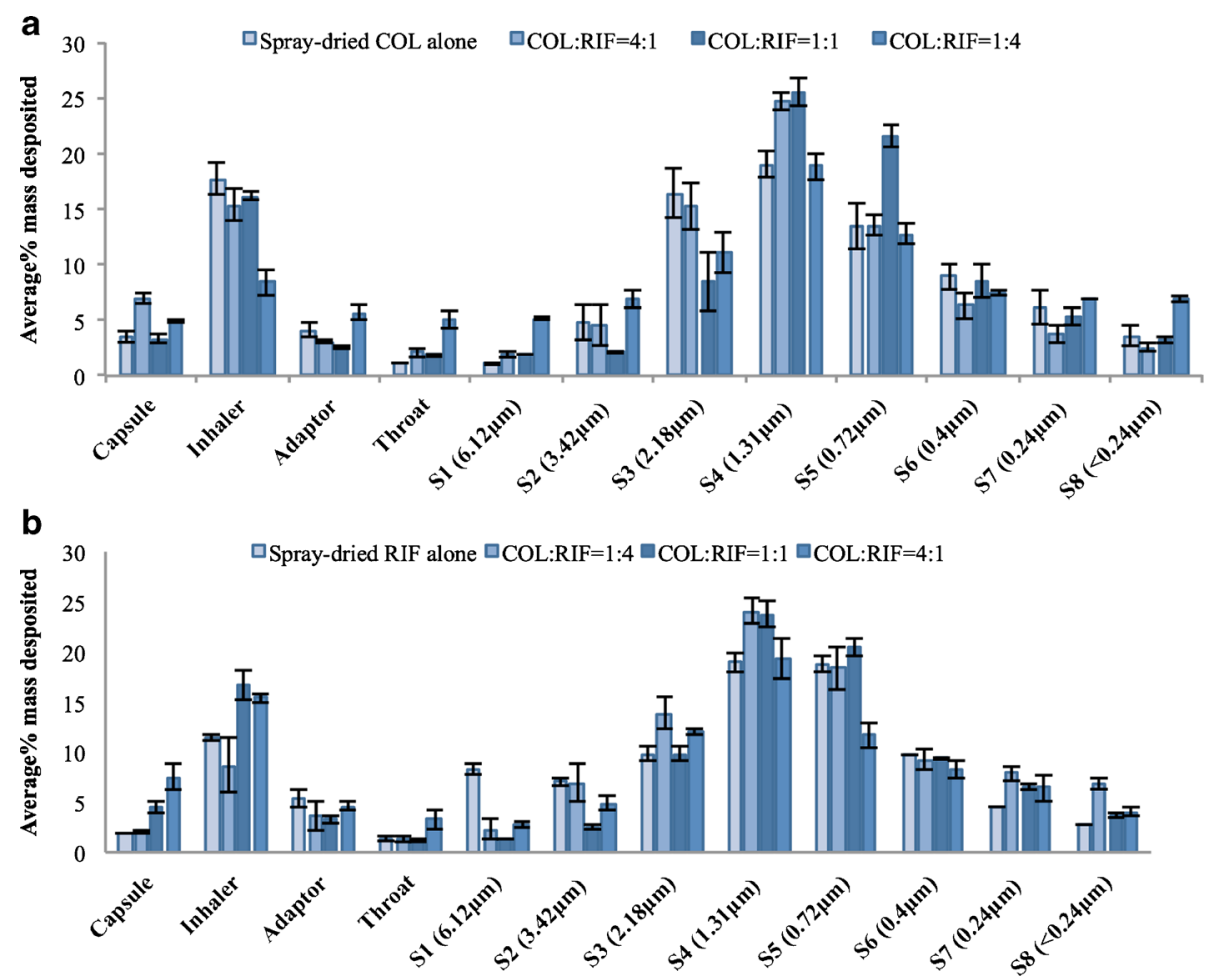

Fig. 4. Drug deposition (a colistin and b rifampicin) of the spray-dried antibiotic powder formulations from the Osmohaler device (error bars represent standard deviation, $n=3$ )

drug load on the dissolution rate measured by the modified Franz cell method: high dose at approximately $3.0 \mathrm{mg}$ and low dose at approximately $1.5 \mathrm{mg}$. The results are shown in Supplement 7. There was no difference in dissolution rate between two doses for both drugs, which indicates the modified Franz cell method is independent of the drug loading, at least for the doses tested here.

\section{Effect of the Drug Ratio on the Dissolution Rate}

The modified Franz cell method was used to investigate the dissolution rate of five different formulations (Fig. 6). The dissolution results in the first $50 \mathrm{~min}$ are given in Supplement 8. There was no significant difference in the dissolution rate for both colistin and rifampicin between different formulations. There was about $20 \%$ of colistin released in $5 \mathrm{~min}$ and around $90 \%$ of colistin dissolved in $25 \mathrm{~min}$. While almost no rifampicin was released in $5 \mathrm{~min}$, approximately $90 \%$ of rifampicin was released in $45 \mathrm{~min}$. The different dissolution profiles of the two drugs can be attributed to the higher solubility of colistin $(50 \mathrm{mg} / \mathrm{mL}$, soluble) (36) than rifampicin (9.9 mg/mL, slightly soluble) $(36,37)$.

\section{DISCUSSION}

Colistin is used as the last-line therapy for respiratory infections (20); however, increasing colistin resistance has been reported in a number of countries (38). The use of combinations of antibiotics is well accepted in clinical practice to enhance the antibacterial efficacy and overcome the bacterial resistance (39). Rifampicin has been frequently used with colistin by parenteral administration and this combination therapy is well tolerated by patients (40). The MIC data showed the combination formulations could provide enhanced antimicrobial effects of colistin against $P$. aeruginosa (Table I), indicating the combination of these two antibiotics may lead to better therapeutic efficacy with lower therapeutic doses, therefore minimising the systemic side effects for the treatment of respiratory infections (41).

Spray drying is a popular approach to produce inhalable particles, attributable to its ability to engineer particles (13). In this study, a co-solvent system of water and ethanol was used to dissolve both water-soluble and water-insoluble drugs for spray drying. Since rifampicin has a lower solubility in the water and co-solvent, it precipitates first on the particle surface during the spray drying process. Consequently, the surfaces of composite particles were dominated by rifampicin ( $87 \%$ of surface was rifampicin) even at a drug ratio of COL/ RIF $=4: 1$, which was confirmed by XPS and ToF-SIMS results (Table II and Fig. 3a). Further increase of rifampicin in the formulation to $80 \%$ resulted in a surface coverage of $98 \%$, suggesting the increased thickness of rifampicin coating. As rifampicin is less hygroscopic than colistin, the combination powders absorbed less water when exposed to the moisture as demonstrated by DVS results. It is interesting to note the change in coating coverage of rifampicin on the particle surfaces for three combination formulations had no significant effects on the aerosol performance. This may be due to the similar aerosol performance of pure colistin and pure rifampicin (Table IV). Overall, all the formulations exhibited relatively high FPF over $64 \%$ (42). Of particular note, a low deposition of the powder in the throat $(<5 \%)$ could lead to 

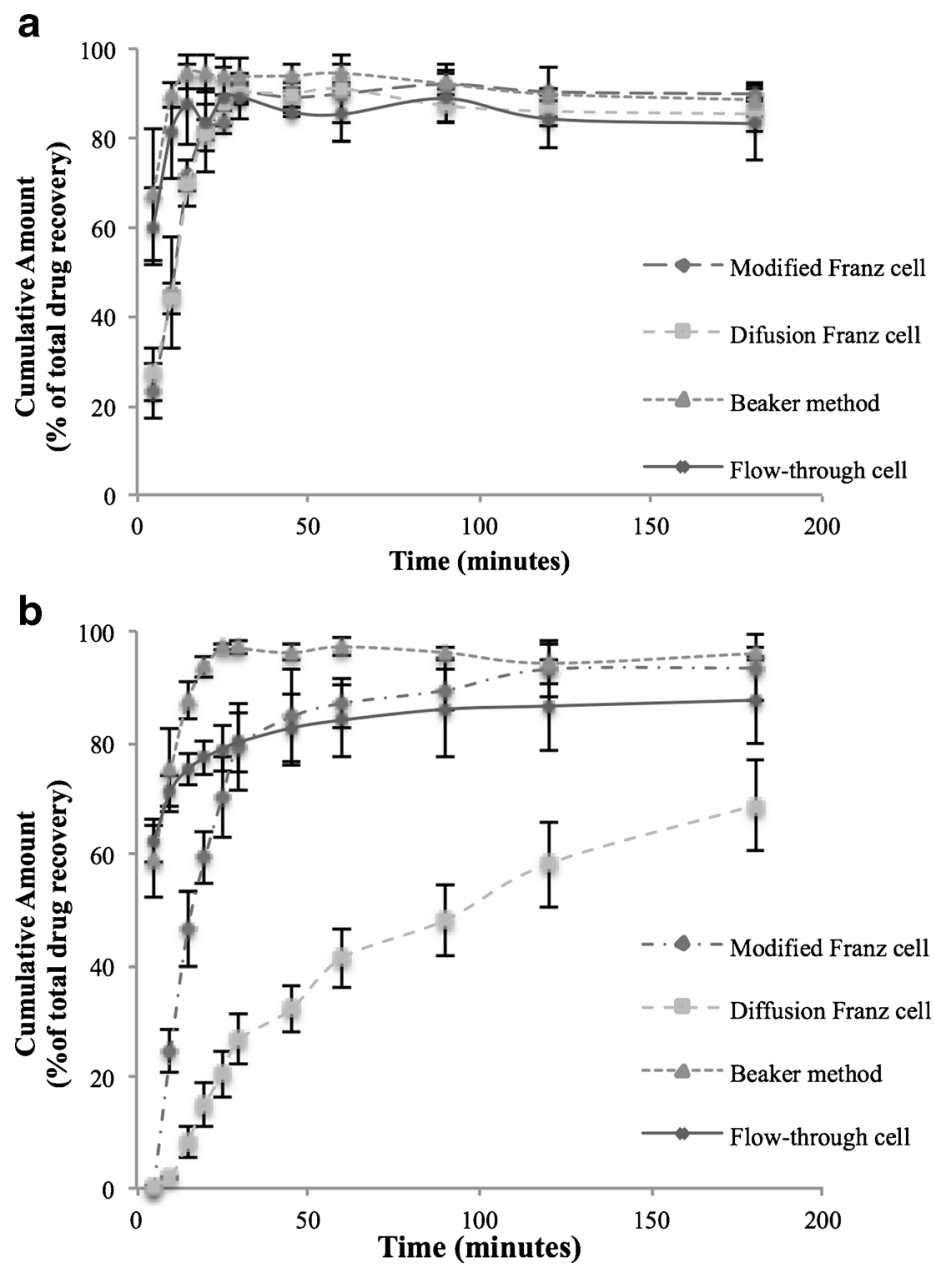

Fig. 5. Different dissolution methods results of a colistin and $\mathbf{b}$ rifampicin (error bars represent standard deviation, $n=3$ )

minimised throat irritation, which is one of the most common side effects after inhalation of colistin. It was noted that the FPF of colistin was lower than that of rifampicin for the formulation of $\mathrm{COL} / \mathrm{RIF}=1: 4$, which was also observed in the previous study of triple antibiotic formulation containing rifampicin, pyrazinamide and isoniazid (5). Higher percentages of drug deposition in the capsule, inhaler and throat were measured for colistin than rifampicin. This phenomenon was only observed for the formulation with a high ratio of rifampicin, which had a high coverage of rifampicin (approximately $98 \%$ ) and a thicker rifampicin coating on the surface. One possible reason could be the outcome of fragmentation of the particle surface (rifampicin). This fragmentation could be due to attrition of particle surface during aerosolisation and/or particle fracture, although the fracture of such fine particles unlikely occurred (particles were small and not crystalline). Such submicron-sized fragments of rifampicin may deposit in the lower stages of NGI (5). This scope of this study does not allow a full investigation into this hypothesis but future studies are warranted to examine the true mechanisms.

Dissolution behaviour is important for a pharmaceutical product since it may correlate with the pharmacokinetics, pharmacodynamics and clinical efficacy (32). Dissolution testing for oral dosage forms has well established; however, there is no standard dissolution testing method for inhaled dry powder formulations (32). Since inhalable antibiotics are supposed to dissolve in the epithelium lining fluid in airways and kill the bacteria, the dissolution profile of inhaled powders in airways may play a vital role in in vivo efficacy, particularly for less water-soluble drugs. In this study, four dissolution methods were investigated and compared for inhalable dry powder of antibiotics.

For both drugs, faster dissolution profiles were evident as measured by the beaker and flow-through cell methods. In the flow-through cell system, a sink condition could be achieved by pumping the continuous flow of fresh or low concentration of the drug medium and the system is very suitable to study the dissolution profile of drugs with a low solubility (43). The faster dissolution is likely because the drug was dissolved in the fresh or low concentration dissolution medium, which was continuously pumped into the cell. The lower total drug release (less than $90 \%$ for both drugs) from flow-through cell method was possibly attributed to the flat geometry of the filter, which potentially generated a high fluid velocity at the center with a decreasing flow gradient towards the periphery (32). This phenomenon leads to the diffusion effect and local non-sink 

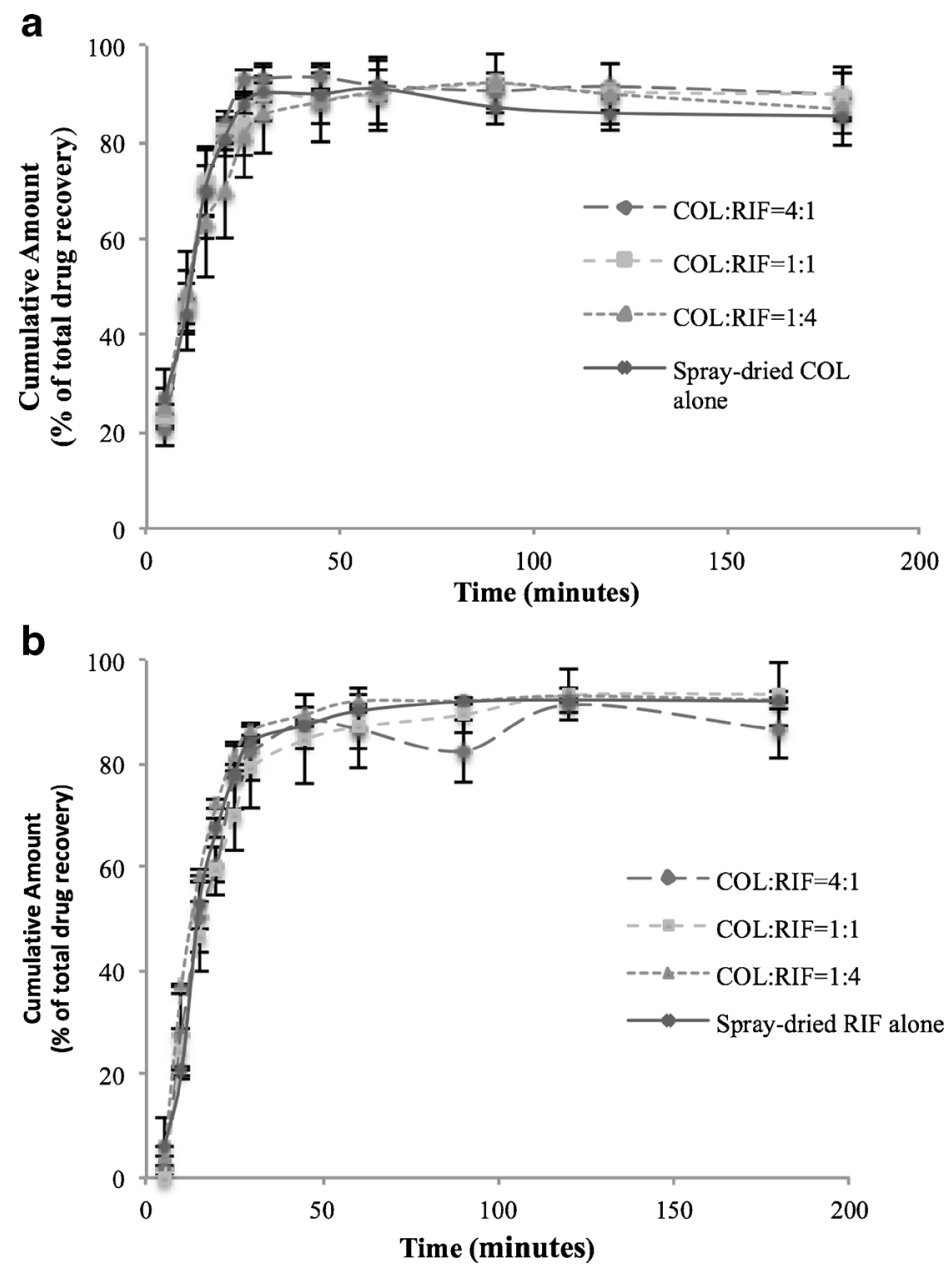

Fig. 6. Dissolution profiles of the spray-dried antibiotic powder formulations measured by the modified Franz cell method (a colistin and $\mathbf{b}$ rifampicin) (error bars represent standard deviation, $n=3$ )

conditions causing the relatively lower dissolution rate in the periphery part (32), as supported by the presence of undissolved powders in the peripheral part after $3 \mathrm{~h}$. The reason for the faster dissolution results in the beaker method was most likely due to the agitation mechanism. Because of the lower dissolution medium height (about $2 \mathrm{~cm}$ ) of the beaker method compared to the Franz cell system (about $5 \mathrm{~cm}$ ), the agitation caused by the stirrer played an important role during the dissolution of drugs. No dissolution difference was measured between the two Franz cell methods for colistin, a water-soluble compound. However, for rifampicin, diffusion Franz cell method led to a lower dissolution rate than the modified Franz cell method and only around $70 \%$ of the total drug was dissolved in $3 \mathrm{~h}$. The reason might because although there was a thin layer of rifampicin contacted with medium on the membrane surface; however, other powder could not be sufficiently wet by the medium thus resulted in a low dissolution rate. Moreover, rifampicin has a high binding effect (44) and the binding to the membrane may decrease the transport rate of drug cross the filter. Compared to rifampicin, colistin is much more hygroscopic (as measured by DVS), and then the indirect contact with medium could still wet all powder sufficiently.
Franz diffusion cell is a dissolution method that has been investigated for evaluating inhaled formulations $(5,45,46)$. However, the membrane may have significant influence on the dissolution profiles measured by the Franz diffusion cell method, particularly for those water insoluble drugs because of insufficient wetting of the powder (32). This study has demonstrated for the hygroscopic colistin, membrane diffusion has negligible effects on the dissolution rate, while for the hydrophobic rifampicin, $30 \%$ drug remained on the top of membrane after $3 \mathrm{~h}$ because of insufficient wetting. However, such a phenomenon of membrane diffusion does not necessarily occur in the lungs in vivo. In order to minimise the artificial impact from the membrane diffusion, the standard Franz cell system was modified, such that the membrane side with the collected powders was placed in contact with the medium. Different drug loadings were investigated for the modified Franz cell method and no difference was found. In future studies, more operation parameters such as medium volume and stirring speed will be evaluated.

The modified Franz cell method was used here to investigate the dissolution rate of five formulations with various drug ratios. The dissolution rate of rifampicin was generally lower than that of colistin because of its lower aqueous solubility and hydrophobic nature. Rifampicin in the 
spray-dried formulations had a relatively fast dissolution rate albeit its hydrophobicity. This could be because all the formulations after spray drying were amorphous, which generally have faster dissolution than the crystalline counterpart as the molecules are randomly arranged in the amorphous structure (47). It is surprising that there was no difference in the dissolution profile of colistin between the pure and combination formulations, despite the surfaces of composite particles being covered by the hydrophobic rifampicin. We hypothesise that the coverage of rifampicin was incomplete, as confirmed by the ToF-SIMS and XPS results. As colistin is present on the composite particle surface, the medium could dissolve the colistin on the surfaces and create tunnels towards the core. Through these tunnels, medium could enter the core of the particle and dissolve colistin in the core. Advanced surface characterisation techniques of ToF-SIMS and XPS are shown to be essential to understand the effects of surface coating on dissolution of composite particles.

\section{CONCLUSIONS}

Five spray-dried inhalable powder formulations with various compositions of colistin and rifampicin were prepared and evaluated. All five spray-dried formulations exhibited relatively high emitted dose (over 77\%) and satisfactory FPF (over $64 \%$ ). The antibacterial activity of colistin against $P$. aeruginosa was enhanced with a mass ratio $(w / w)$ of colistin to rifampicin of $4: 1$, though the formulation of COL/ RIF 1:4 had higher emitted dose for both drugs and higher FPF of rifampicin. Hydrophobic nature of rifampicin resulted in a surface enrichment on the co-spray dried particles as indicated by XPS and ToF-SIMS data. DVS results suggested that the surface enrichment of rifampicin on the particle surfaces could suppress the moisture sorption of the spraydried powder, whereas the surface enrichment of hydrophobic rifampicin did not result in any delay in dissolution rate of colistin in different formulations because the coverage of rifampicin on the particle surface is incomplete. In this study, the advanced surface characterisation techniques of XPS and ToF-SIMS have been exploited for the first time to understand the effect of surface composition on the aerosolisation and dissolution behaviour of spray-dried combination powder formulations.

\section{ACKNOWLEDGMENTS}

The authors acknowledge the financial support from the Australian Research Council's Discovery Project funding scheme (DP120102778) and the National Health and Medical Research Council's (NHMRC) Project Grant funding scheme (APP1065046). Jian Li is an NHMRC Senior Research Fellow and his research is also supported by grants from the National Institute of Allergy and Infectious Diseases of the National Institutes of Health (R01 A1098771 and R01 AI111965). The content is solely the responsibility of the authors and does not necessarily represent the official views of the National Institute of Allergy and Infectious Diseases or the National Institutes of Health. The authors are grateful for the scientific and technical assistance, of the Australian Microscopy \& Microanalysis Research Facility at the
Australian Centre for Microscopy and Microanalysis, The University of Sydney and the Ian Wark Research Institute, University of South Australia. The authors also acknowledge the International office and the School of Pharmaceutical Sciences of University of Copenhagen for the financial support to Wenbo Wang's stay in Sydney.

\section{REFERENCES}

1. WHO. The 10 leading causes of death in the world, 2000 and 2012. who.int/mediacentre/factsheets/fs310/en/. May 2014.

2. Velkov T, Rahim NA, Zhou QT, Chan H-K, Li J. Inhaled antiinfective chemotherapy for respiratory tract infections: successes, challenges and the road ahead. Adv Drug Deliv Rev. 2015;85:65-82.

3. Garonzik SM, Li J, Thamlikitkul V, Paterson DL, Shoham S, $\mathrm{Jacob} \mathrm{J}$, et al. Population pharmacokinetics of colistin methanesulfonate and formed colistin in critically ill patients from a multicenter study provide dosing suggestions for various categories of patients. Antimicrob Agents Chemother. 2011;55(7):3284-94.

4. Zhou Q, Leung SSY, Tang P, Thaigarajan P, Loh Z, Chan HK. Inhalation formulation and drug delivery systems for respiratory infections. Adv Drug Deliv Rev. 2015;85:83-99.

5. Chan JGY, Chan H-K, Prestidge CA, Denman JA, Young PM, Traini D. A novel dry powder inhalable formulation incorporating three first-line anti-tubercular antibiotics. Eur J Pharm Biopharm. 2013;83(2):285-92.

6. Zhou QT, Gengenbach T, Denman JA, Heidi HY, Li J, Chan HK. Synergistic antibiotic combination powders of colistin and rifampicin provide high aerosolization efficiency and moisture protection. AAPS J. 2014;16(1):37-47.

7. Yapa SWS, Li J, Patel K, Wilson JW, Dooley MJ, George J, et al. Pulmonary and systemic pharmacokinetics of inhaled and intravenous colistin methanesulfonate in cystic fibrosis patients: targeting advantage of inhalational administration. Antimicrob Agents Chemother. 2014;58(5):2570-9.

8. Döring G, Flume P, Heijerman H, Elborn JS, Study G. Consensus. Treatment of lung infection in patients with cystic fibrosis: current and future strategies. J Cyst Fibros. 2012;11(6):461-79.

9. Zhou QT, Tang P, Leung SSY, Chan JGY, Chan H-K. Emerging inhalation aerosol devices and strategies: where are we headed? Adv Drug Deliv Rev. 2014;75:3-17.

10. Melani AS, Zanchetta D, Barbato N, Sestini P, Cinti C, Canessa $\mathrm{PA}$, et al. Inhalation technique and variables associated with misuse of conventional metered-dose inhalers and newer dry powder inhalers in experienced adults. Ann Allergy Asthma Immunol. 2004;93(5):439-46.

11. Lavorini F, Magnan A, Dubus JC, Voshaar T, Corbetta L, Broeders M, et al. Effect of incorrect use of dry powder inhalers on management of patients with asthma and COPD. Respir Med. 2008;102(4):593-604.

12. Depreter F, Pilcer G, Amighi K. Inhaled proteins: challenges and perspectives. Int J Pharm. 2013;447(1):251-80.

13. Seville PC, Li H-y, Learoyd TP. Spray-dried powders for pulmonary drug delivery. Crit Rev' ${ }^{\mathrm{TM}}$ Ther Drug Carrier Syst. 2007;24(4).

14. Sou T, Kaminskas LM, Nguyen TH, Carlberg R, McIntosh MP, Morton DAV. The effect of amino acid excipients on morphology and solid-state properties of multi-component spray-dried formulations for pulmonary delivery of biomacromolecules. Eur J Pharm Biopharm. 2013;83(2):234-43.

15. Sou T, Orlando L, McIntosh MP, Kaminskas LM, Morton DAV. Investigating the interactions of amino acid components on a mannitol-based spray-dried powder formulation for pulmonary delivery: a design of experiment approach. Int J Pharm. 2011;421(2):220-9.

16. Kwok PCL, Tunsirikongkon A, Glover W, Chan H-K. Formation of protein nano-matrix particles with controlled surface architecture for respiratory drug delivery. Pharm Res. 2011;28(4):788-96. 
17. Haynes A, Nakamura J, Heng C, Heuerding S, Thompson G, Malcolmson R, editors. Aerosol performance of tobramycin inhalation powder. Respiratory Drug Delivery; 2010.

18. Zhou Q, Morton D, Yu HH, Jacob J, Wang JP, Li J, et al. Colistin powders with high aerosolisation efficiency for respiratory infection: preparation and in vitro evaluation. J Pharm Sci. 2013;102(10):3736-47.

19. Zhou QT, Sun S-P, Chan JGY, Wang P, Barraud N, Rice SA, et al. A novel inhaled combination powder containing amorphous colistin and crystalline rifapentine with enhanced antimicrobial activities against planktonic cells and biofilm of Pseudomonas aeruginosa for respiratory infections. Mol Pharm. 2015;12(8):2594-603

20. Li J, Nation RL, Milne RW, Turnidge JD, Coulthard K. Evaluation of colistin as an agent against multi-resistant Gramnegative bacteria. Int J Antimicrob Agents. 2005;25(1):11-25.

21. Barraud N, Buson A, Jarolimek W, Rice SA. Mannitol enhances antibiotic sensitivity of persister bacteria in Pseudomonas aeruginosa biofilms. PLOS ONE. 2013;8(12).

22. Wan F, Bohr A, Maltesen MJ, Bjerregaard S, Foged C, Rantanen $\mathrm{J}$, et al. Critical solvent properties affecting the particle formation process and characteristics of celecoxib-loaded PLGA microparticles via spray-drying. Pharm Res. 2013;30(4):1065-76.

23. Shekunov BY, Chattopadhyay P, Tong HHY, Chow AHL. Particle size analysis in pharmaceutics: principles, methods and applications. Pharm Res. 2007;24(2):203-27.

24. Zhou QT, Denman JA, Gengenbach T, Das S, Qu L, Zhang H, et al. Characterization of the surface properties of a model pharmaceutical fine powder modified with a pharmaceutical lubricant to improve flow via a mechanical dry coating approach. J Pharm Sci. 2011;100(8):3421-30.

25. Beamson G, Briggs D. High resolution XPS of organic polymers. 1st ed. Chichester: Wiley; 1992.

26. Asking L, Olsson B. Calibration at different flow rates of a multistage liquid impinger. Aerosol Sci Technol. 1997;27(1):39-49.

27. Pilcer G, Vanderbist F, Amighi K. Spray-dried carrier-free dry powder tobramycin formulations with improved dispersion properties. J Pharm Sci. 2009;98(4):1463-75.

28. Duport P, Robertson R, Ho K, Horvath F. Flow-through dissolution of uranium-thorium ore dust, uranium concentrate, uranium dioxide, and thorium alloy in simulated lung fluid. Radiat Prot Dosim. 1991;38(1-3):121-33.

29. Rennard S, Basset G, Lecossier D, O'Donnell KM, Pinkston P, Martin $\mathrm{P}$, et al. Estimation of volume of epithelial lining fluid recovered by lavage using urea as marker of dilution. J Appl Physiol. 1986;60(2):532-8.

30. O'Hara P, Hickey AJ. Respirable PLGA microspheres containing rifampicin for the treatment of tuberculosis: manufacture and characterization. Pharm Res. 2000;17(8):955-61.

31. Heng D, Cutler DJ, Chan H-K, Yun J, Raper JA. What is a suitable dissolution method for drug nanoparticles? Pharm Res. 2008;25(7):1696-701.

32. Riley T, Christopher D, Arp J, Casazza A, Colombani A, Cooper $\mathrm{A}$, et al. Challenges with developing in vitro dissolution tests for orally inhaled products (OIPs). AAPS PharmSciTech. 2012;13(3):978-89.
33. York P. Analysis of moisture sorption hysteresis in hard gelatin capsules, maize starch, and maize starch: drug powder mixtures. J Pharm Pharmacol. 1981;33(1):269-73.

34. Piao H, McIntyre N. Adventitious carbon growth on aluminium and gold-aluminium alloy surfaces. Surf Interface Anal. 2002;33(7):591-4.

35. Coates MS, Fletcher DF, Chan H-K, Raper JA. The role of capsule on the performance of a dry powder inhaler using computational and experimental analyses. Pharm Res. 2005;22(6):923-32.

36. Notices G. 1.4 Monographs European pharmacopoeia. European Directorate for the Quality of Medicines and HealthCare, Strasbourg; 2010.

37. Henwood SQ, Liebenberg W, Tiedt LR, Lötter AP, de Villiers MM. Characterization of the solubility and dissolution properties of several new rifampicin polymorphs, solvates, and hydrates. Drug Dev Ind Pharm. 2001;27(10):1017-30.

38. Li J, Nation RL, Turnidge JD, Milne RW, Coulthard K, Rayner $\mathrm{CR}$, et al. Colistin: the re-emerging antibiotic for multidrugresistant Gram-negative bacterial infections. Lancet Infect Dis. 2006;6(9):589-601.

39. Lee SH, Teo J, Heng D, Ng WK, Chan H-K, Tan RBH. Synergistic combination dry powders for inhaled antimicrobial therapy: formulation, characterization and in vitro evaluation. Eur J Pharm Biopharm. 2013;83(2):275-84.

40. Song JY, Lee J, Heo JY, Noh JY, Kim WJ, Cheong HJ, et al. Colistin and rifampicin combination in the treatment of ventilator-associated pneumonia caused by carbapenemresistant Acinetobacter baumannii. Int J Antimicrob Agents. 2008;32(3):281-4.

41. Adi H, Young PM, Chan HK, Stewart P, Agus H, Traini D. Cospray dried antibiotics for dry powder lung delivery. J Pharm Sci. 2008;97(8):3356-66.

42. Zhou QT, Armstrong B, Larson I, Stewart PJ, Morton DAV. Understanding the influence of powder flowability, fluidization and de-agglomeration characteristics on the aerosolization of pharmaceutical model powders. Eur J Pharm Sci. 2010;40(5):412-21.

43. Davies NM, Feddah MR. A novel method for assessing dissolution of aerosol inhaler products. Int J Pharm. 2003;255(1):175-87.

44. White RJ, Lancini G. Uptake and binding of $\left[{ }^{3} \mathrm{H}\right]$ rifampicin by Escherichia coli and Staphylococcus aureus. Biochim Biophys Acta (BBA)-Nucleic Acids Protein Synth. 1971;240(3):429-34.

45. May S, Jensen B, Wolkenhauer M, Schneider M, Lehr CM. Dissolution techniques for in vitro testing of dry powders for inhalation. Pharm Res. 2012;29(8):2157-66.

46. Salama RO, Traini D, Chan H-K, Young PM. Preparation and characterisation of controlled release co-spray dried drugpolymer microparticles for inhalation 2: evaluation of in vitro release profiling methodologies for controlled release respiratory aerosols. Eur J Pharm Biopharm. 2008;70(1):145-52.

47. Morris KR, Griesser UJ, Eckhardt CJ, Stowell JG. Theoretical approaches to physical transformations of active pharmaceutical ingredients during manufacturing processes. Adv Drug Deliv Rev. 2001;48(1):91-114. 